\title{
Teeth Extractions in Subjects Undergoing Radiotherapy for Head and Neck Cancers: A Systematic Review on the Clinical Protocols for Preventing Osteoradionecrosis (ORN). Extractions after Radiotherapy (Part 2) ${ }^{\dagger}$
}

\author{
Gioele Gioco 1,*, Cosimo Rupe 1, Giuseppe Troiano ${ }^{2}$, Michele Giuliani ${ }^{2}$, Massimo Petruzzi ${ }^{3}$ and \\ Carlo Lajolo ${ }^{1}$ \\ 1 Head and Neck Department, “Fondazione Policlinico Universitario A. Gemelli-IRCCS”, School of Dentistry, \\ Università Cattolica del Sacro Cuore, 00168 Rome, Italy; cosimorupe@gmail.com (C.R.); \\ carlo.lajolo@unicatt.it (C.L.) \\ 2 Department of Clinical and Experimental Medicine, University of Foggia, 71122 Foggia, Italy; \\ giuseppe.troiano@unifg.it (G.T.); michele.giuliani@unifg.it (M.G.) \\ 3 Interdisciplinary Department of Medicine, University “Aldo Moro” of Bari, 70121 Bari, Italy; \\ massimo.petruzzi@uniba.it \\ * Correspondence: gioele.gioco@hotmail.it; Tel.: +39-3288486585 \\ + Presented at the XV National and III International Congress of the Italian Society of Oral Pathology and \\ Medicine (SIPMO), Bari, Italy, 17-19 October 2019.
}

Published: 11 December 2019

Osteoradionecrosis (ORN) of the jaws is the most severe side effect, and in some case lifethreatening, of radiotherapy for head and neck cancer [1]. Tooth extractions during and after radiotherapy are the major risk factor of ORN onset, nevertheless the real ORN rate and its risk factors are still unclear. Recently, a systematic review [2] tried to better estimate the ORN rate following tooth extractions after RT, pointing out an overall rate of $7 \%$, nevertheless the high number of variables involved in the onset of ORN makes the topic particularly complex and many information still lack to prevent the ORN onset after tooth extraction. The aim of this systematic review is to assess the incidence of ORN in those patients who underwent tooth extraction during and after radiotherapy for head and neck cancer and to identify any possible risk factors.

PRISMA protocol was used to evaluate and present the results. PubMed, Scopus, and Web of Science were used as search engines: only English full-length papers of clinical trial and observational studies both prospective and retrospective, published in peer-reviewed journals, were investigated. Inclusion criteria were: minimum sample size 10 patients who underwent tooth extractions during and after radiotherapy; 6 months mean follow-up after tooth extraction; no previous ORN at extraction site; the ORN diagnosis criteria must be clearly defined in the text; must be specified if ORN developed at extraction site or not. Cumulative meta-analysis was performed calculating the pooled proportion (PP) of the rate of ORN occurrence. Meta-analysis was performed at random effects model with the Der-Simonian Liard method. All the statistical analyses were performed with the software Open Meta-Analyst version 10. PROSPERO registration code is CRD42018079986.

Among 2020 records screened, only 9 were included in this review (Figure 1). Forty-one of 462 patients who underwent tooth extraction during and after radiotherapy developed ORN at extraction site, with an ORN incidence of 5.8\% (95\% Confidence of Interval $=2.5-9.4, p<0.001, \mathrm{I}^{2}=8322 \%$ ). 


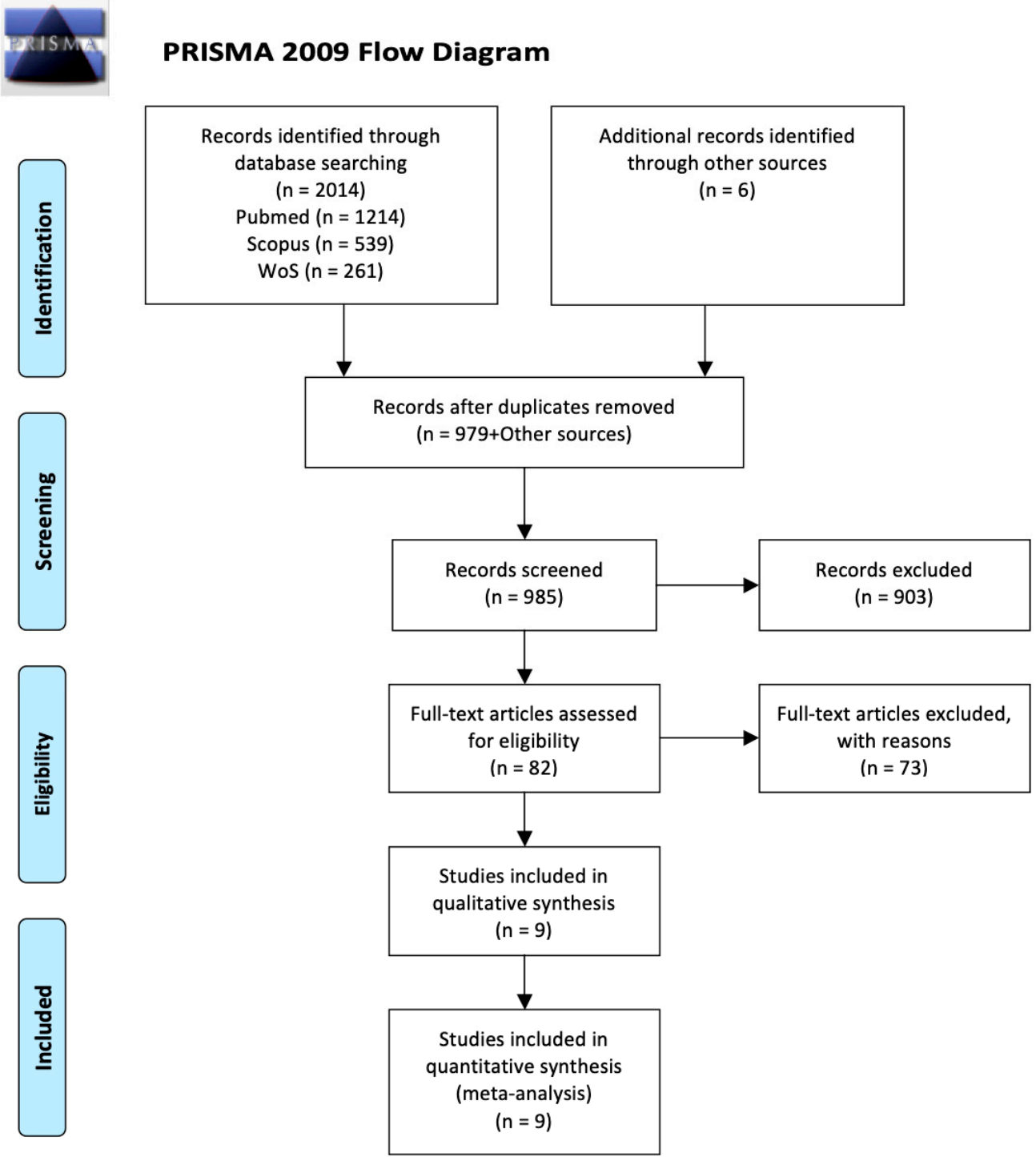

From: Moher D, Liberati A, Tetzlaff J, Altman DG, The PRISMA Group (2009). Preferred Reporting Items for Systematic Reviews and MetaAnalyses: The PRISMA Statement. PLoS Med 6(7): e1000097. doi:10.1371/journal.pmed1000097

For more information, visit www.prisma-statement.org.

Figure 1. PRISMA flow-chart of the inclusion process.

This systematic review confirmed dental extraction after radiotherapy as the main risk factor of ORN and its incidence ranges between $6 \%$ and $11 \%$ (Figure 2). The considerable amount of missing data didn't allow us to identify other possible risk factors for ORN onset. 


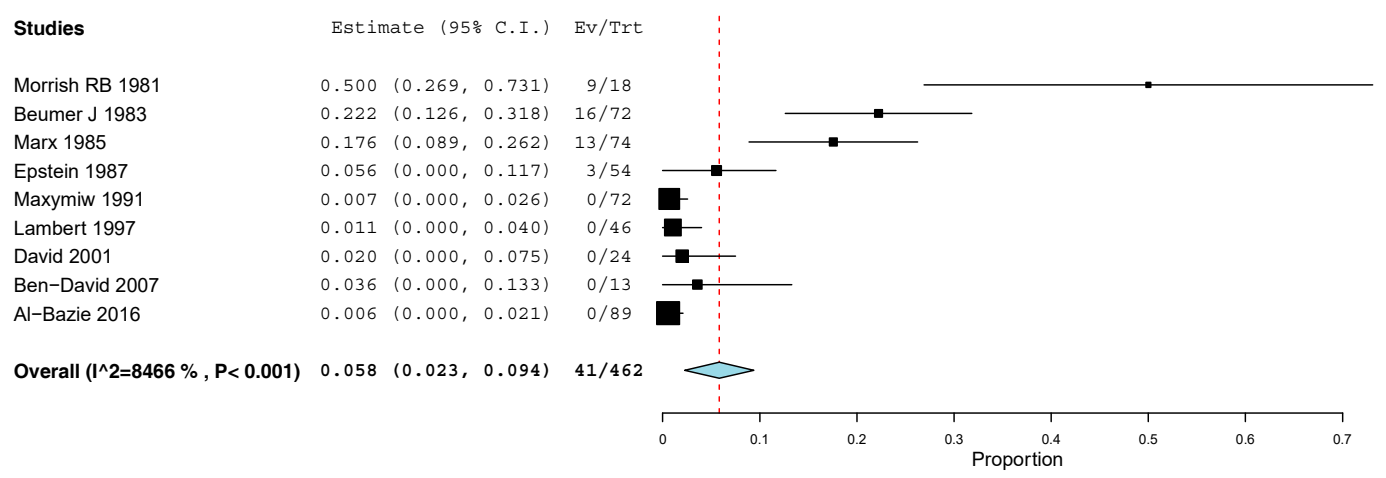

Figure 2. Pooled data and box plot stratifying for pathological criteria.

Although the most recently performed studies highlight a considerable decreasing of ORN incidence, the results confirm the recommendation to perform a dental evaluation and to remove oral foci before the beginning of radiotherapy.

Conflicts of Interest: The authors declare no conflict of interest.

\section{References}

1. Nadella, K.; Kodali, R.; Guttikonda, L.; Jonnalagadda, A. Osteoradionecrosis of the Jaws: ClinicoTherapeutic Management: A Literature Review and Update. J. Maxillofac. Oral Surg. 2015, 14, 891-901.

2. Nabil, S.; Samman, N. Incidence and prevention of osteoradionecrosis after dental extraction in irradiated patients: A systematic review. Int. J. Oral Maxillofac. Surg. 2011, 40, 229-243.

(C) 2019 by the authors. Licensee MDPI, Basel, Switzerland. This article is an open access article distributed under the terms and conditions of the Creative Commons Attribution (CC BY) license (http://creativecommons.org/licenses/by/4.0/). 